\title{
TI.51.1
}

\section{Middleware Integration with Existing Applications: Current Design Issues, with a Focus on Mailing Lists}

- PDF: draft-internet2-mace-mlist-middleware-integration-issues-03.pdf

- HTML: draft-internet2-mace-mlist-middleware-integration-issues-03.html

More Information

\begin{tabular}{|c|c|}
\hline Repository ID & TI.51.1 \\
\hline Persistent URL & http://doi.org/10.26869/TI.51.1 \\
\hline Title & Middleware Integration with Existing Applications: Current Design Issues, with a Focus on Mailing Lists \\
\hline Authors & Jill Gemmill, John-Paul Robinson \\
\hline Sponsor & NMI-Edit \\
\hline \multicolumn{2}{|l|}{ Review } \\
\hline Status & Legacy \\
\hline Publish Date & 2005 \\
\hline DOI & $10.26869 / \mathrm{TI} .51 .1$ \\
\hline \multicolumn{2}{|l|}{ Signature } \\
\hline Deprecated & No \\
\hline \multicolumn{2}{|l|}{ Future Review } \\
\hline \multicolumn{2}{|l|}{ Supersedes } \\
\hline \multicolumn{2}{|l|}{ Format } \\
\hline \multicolumn{2}{|l|}{ Related Docs } \\
\hline \multicolumn{2}{|l|}{ Development Location } \\
\hline \multicolumn{2}{|l|}{ IP Framework } \\
\hline Subject Tags & middlewarerescue \\
\hline Notes & \\
\hline
\end{tabular}

